\title{
Polarization analysis of antireflection coating for SOS material system
}

\author{
Imran Khan, M. Mostafizur Rahman \\ Dept. of Electronics \& Communication Engineering, Khulna University of Engineering \& Technology, Khulna, Bangladesh
}

\author{
Email address: \\ ikr_ece@yahoo.com(I. Khan)
}

\section{To cite this article:}

Imran Khan, M. Mostafizur Rahman. Polarization Analysis of Antireflection Coating for SOS material System. Optics.

Vol. 2, No. 3, 2013, pp. 42-46. doi: 10.11648/j.optics.20130203.11

\begin{abstract}
Using CAD tool Essential Macleod, MATLAB and Optimac-investigation is done on an antireflection coating for SOS material system. Here the polarization (TE \& TM) effect of antireflection coating for SOS material system is mainly analyzed. No coating, single layer coating, optimized single layer coating and finally multilayer coating are used to investigate the polarization effect. It is observed that the mean transmission of $96.56 \%, 98.74 \%$ and $99.99 \%$ obtained for no coating, single layer coating and multilayer coating of a SOS material system respectively.
\end{abstract}

Keywords: Anti Reflection Coating, Silica on Silicon, TM, TE, Polarization

\section{Introduction}

Reflection is the optical phenomenon, which is born out of a transition in the medium, in which light is travelling. Any optical medium is characterized by the parameter known as refractive index (n) and quantifies the speed of light in the current medium with respect to that in vacuum. Normally a fraction of incident light reflected from the interface of an optical medium is measured by reflectance, and the rest transmitted (refracted) is measured by transmittance. The mathematical model to calculate the refractive index of a thin film is [1]-

$$
\begin{aligned}
& n_{A R}=\sqrt{n_{1} n_{2}} \\
& \text { physical thickness }=\frac{1}{4} \cdot \frac{\lambda}{n_{A R}}
\end{aligned}
$$

Where, $\mathrm{n}_{\mathrm{AR}}$ is the refractive index of the thin film, $\mathrm{n}_{1} \& \mathrm{n}_{2}$ are the refractive index of the related two mediums and $\lambda$ is the wavelength of the light.

The first material system applied for fabricating commercial photonic integrated circuits for telecom applications on silicon was silica. Thanks to the very low loss of straight waveguides $(0.03 \mathrm{~dB} / \mathrm{cm})$ at a wavelength of $\lambda=1550 \mathrm{~nm}$. The downside of this material system however is the low refractive index contrast of this material which implies huge bends.
To fabricate a waveguide in silica on silicon (Fig.1) is done by heavily doping a $\mathrm{SiO}_{2}$ layer with germanium. This results a refractive index difference of about $0.75 \%$ with the undoped $\mathrm{SiO}_{2}$. A waveguide is defined in the doped $\mathrm{SiO}_{2}$ through an etching process, and afterwards an

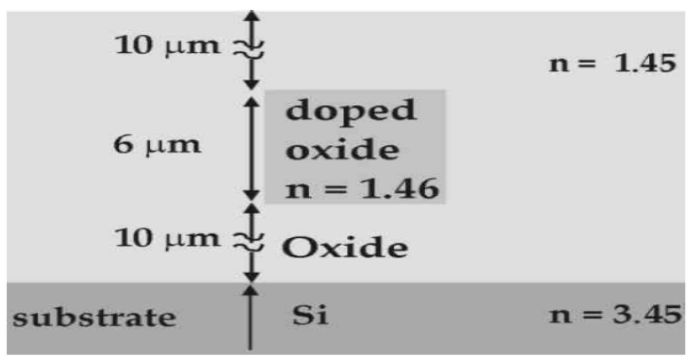

Figure 1. Cross-section of a waveguide fabricated in silica on silicon.

undoped $\mathrm{SiO}_{2}$ cladding layer is deposited. Anti reflection coatings (ARC) are designed for different purposes [2], but here we considered the analysis to get a general polarization characteristic of the ARC.

We analyzed the transmission of the guided mode of the waveguide structure through the interface between the photonic integrated circuit and its surrounding medium (air). We wanted to maximize the transmission, to achieve this we used a single-layer coating, which we then optimized and finally we designed a multi-layer coating with the aid of the CAD tool Essential Macleod. We analyzed the transmission for Silica on Silicon (SOS) material system. In Essential Macleod we can only calculate the transmission through a 
stack of homogeneous layers [3],[4] so we modelled the cross section of the waveguide structure by one single number, the effective index $n_{\text {eff }}$ of the guided mode of the structure. The transmittance can be increased using the ARC as explained in [8].

\section{Simulation}

By using the CAD software we can calculate the transmission of plane wave incident at an arbitrary angle. But the guided modes of the waveguide structures are approximated by Gaussian beam. The properties of this Gaussian beam are given in Table I. We solved this problem by decomposing the Gaussian beam into its plane wave components using the spatial Fourier Transform, calculating the transmission for each of these components. Afterwards using the inverse Fourier transform we obtain the transmitted beam.

Table 1. Dimensions of the Gaussian field profile.

\begin{tabular}{lllll}
\hline Material System & $\boldsymbol{\lambda}_{\mathbf{0}}[\boldsymbol{\mu \mathrm { m } ]}$ & $\boldsymbol{\sigma}_{\mathrm{x}}[\boldsymbol{\mu m}]$ & $\boldsymbol{\sigma}_{\mathrm{y}}[\boldsymbol{\mu m}]$ & $\mathbf{n}_{\left(\mathbf{n}_{\mathrm{eff}}\right)}$ \\
\hline Silica on Silicon (SOS) & 1.55 & 3.63 & 3.63 & 1.455 \\
\hline
\end{tabular}

In Essential Macleod we calculated the transmission through a layer stack. This was built up of a semi-infinite substrate layer (in this case the waveguide), a number of thin films and another semi-infinite medium (in this case air). The thickness of each layer can be set by a physical thickness and by an optical thickness. The optical thickness is related with the wavelength and refractive index as-

$$
\text { Optical thickness }=(\text { Physical thickness }) /(\lambda / \mathrm{n})
$$

\section{Findings \& Analysis}

\subsection{Without Coating}

First we considered the transmission of light for the SOS material system without any AR coating. This transmission was conducted to consider it as the reference. The input field amplitude is shown in Fig. 3. This input field was used throughout the whole simulation, so that we can compare among all the results easily and effectively. Fig.4 represents the mean output field amplitude.

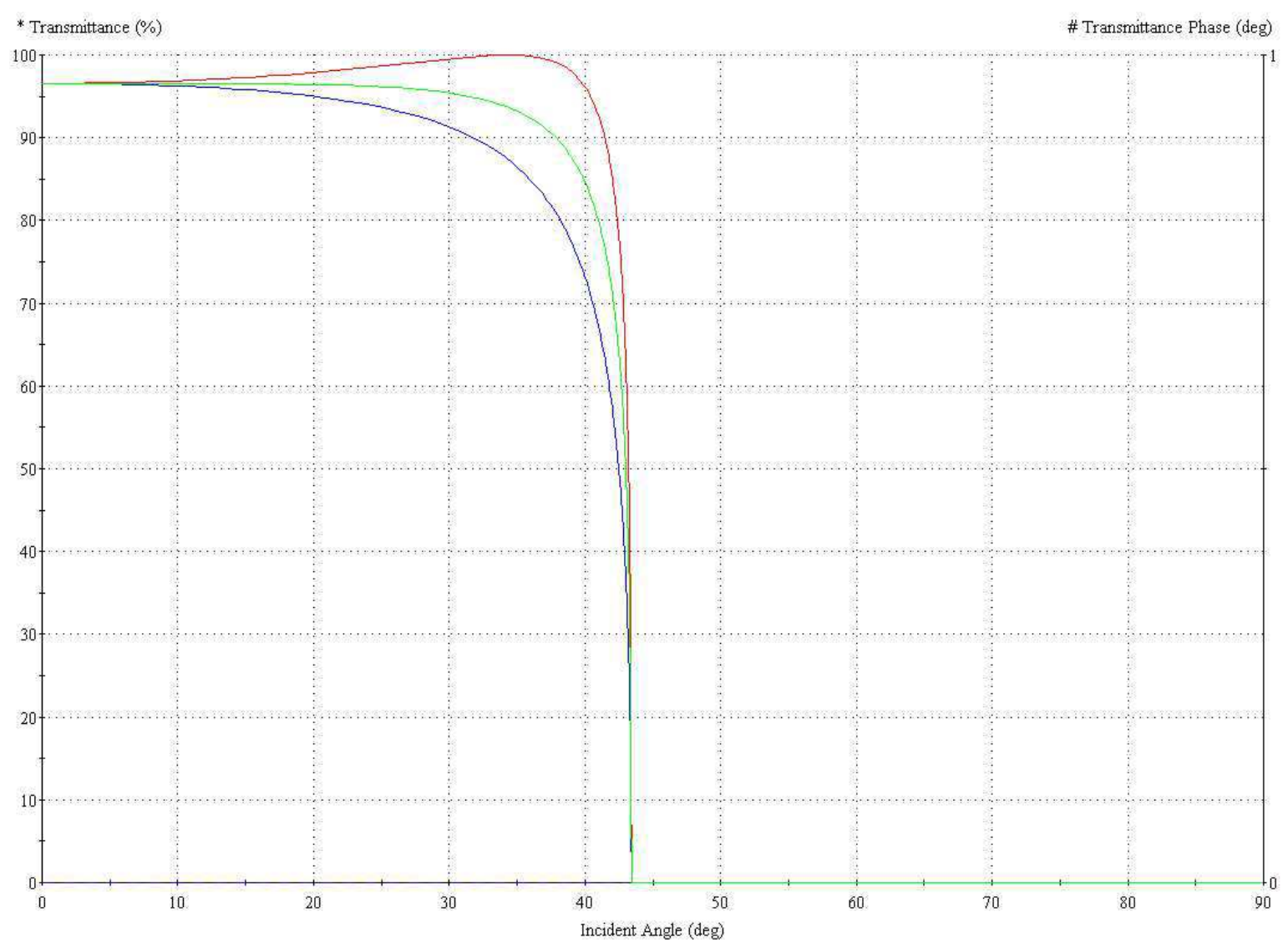

Figure 2. Transmission for all polarisations when there is no coating: magnitude (solid lines) and phase (dashed lines). TM: Red, TE: Blue, Mean: Green. As there is no phase change so the dashed lines coincides with the incident angle axis. 


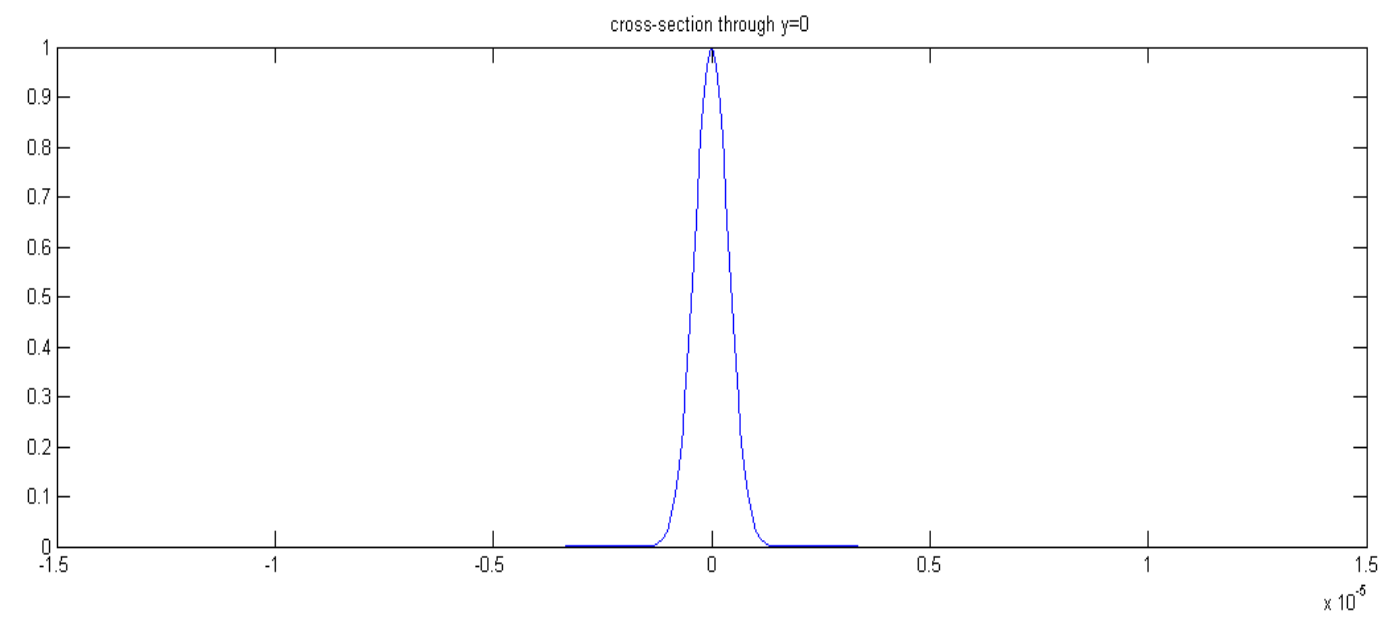

Figure 3. Input field amplitude (cross-section through $y=0$, assuming arbitrary units along $x$ and $y$ axis).

Without any coating the critical angle was found as $43.42^{\circ}$. Angle consideration of ARC for the telecom wavelength can be found in [10]. It is clear from the Fig.2 that there is no transmission above the critical angle. We also noticed, there is $100 \%$ transmission for TM-polarisation at Brewster angle [11]. i.e.-

$$
\text { Brewster angle }=\tan ^{-1}\left(\frac{1}{1.455}\right)=34.50^{\circ}
$$

It is observed that the calculated and the simulated Brewster angle is the same. Next we considered the transmission for incident angle $=0^{\circ}$ (perpendicular incidence):

$$
\begin{aligned}
& r=\frac{n_{\text {eff }}-1}{n_{\text {eff }}+1}=0.19 \\
& T=1-R=1-r^{2}=97 \%
\end{aligned}
$$

So the transmission for perpendicular incidence was $97 \%$ and this also matched with the simulated result as shown in Fig.2. In this case the phase is zero everywhere in the Fig.2. There was no coating so no phase shift occurred for the transmission. This results no interference. Because of the Brewster angle the TE-transmission is slightly lower than TM-transmission but has similar amplitude profile. No interference fringes around the edges of the Gaussian beam were observed because the beam width was large compared to the wavelength.

Global transmission was calculated using the Table-1 parameters and MATLAB simulator.

Global transmission:

\begin{tabular}{lll}
\hline TM Polarized light & TE Polarized light & Mean \\
\hline 96.5871 & 96.5428 & 96.5650 \\
\hline
\end{tabular}

As expected, the mean value is slightly lower than the value for perpendicular incidence because the Gaussian beam has also plane wave components with a more oblique incidence (referring to Fig.2).

\subsection{Single Layer Coating}

Using (1) we can get the theoretical values for single layer coating:

$$
n_{A R}=\sqrt{1.455}=1.206
$$

We considered a material with $\mathrm{n}$ close to this value$\mathrm{Na}_{3} \mathrm{AlF}_{6}: \mathrm{n}$ ' $=1.35 ; \mathrm{n}$ " $=0$ (n' for real; n" for imaginary)

Results from the simulation were as follows-

Critical angle when $\theta_{\text {air }}=90^{\circ}$ or $\theta_{\mathrm{AR}}=90^{\circ}$ :

$$
\theta_{S O S c \text { crit }}=\min \left\{\arcsin \left(\frac{n_{\text {air }}}{n_{\text {SOS }}}\right), \arcsin \left(\frac{n_{A R}}{n_{\text {SOS }}}\right)\right\}=\arcsin \left(\frac{n_{\text {air }}}{n_{\text {SOS }}}\right)=43.42^{\circ}(6)
$$

Phase change was different for TE \& TM polarization. The phase change is observed due to the interference of the different plane wave components of the Gaussian beam. This results a lower transmission.

TM-transmission was slightly higher than the TE-transmission because of smaller phase shift for TM than for TE (referring to Fig.5). i.e. less interference of the plane waves.

Global transmission:

\begin{tabular}{ccc}
\hline TM Polarized light & TE Polarized light & Mean \\
\hline 98.7524 & 98.7329 & 98.7427 \\
\hline
\end{tabular}

These transmission values are better than the ones for silicon on insulator (SOI) [5]. This is because the beam width of the Gaussian beam relatively larger compared to the wavelength, which results the plane wave decomposition spread out. The critical angle was also much larger than for SOI. So less plane wave components were completely lost. Single layer reflectance can also be calculated using [6] for comparison purposes. The result shown in Fig. 5 can be compared with the reflectance in [7][12]. 


\subsection{Optimized Single Layer Coating}

We optimized the transmission through the coating by changing the thickness of the layer using Simplex. The op- timization leads to a thickness very close to the theoretical value, and there was no difference in the transmission values of the Gaussian beam.

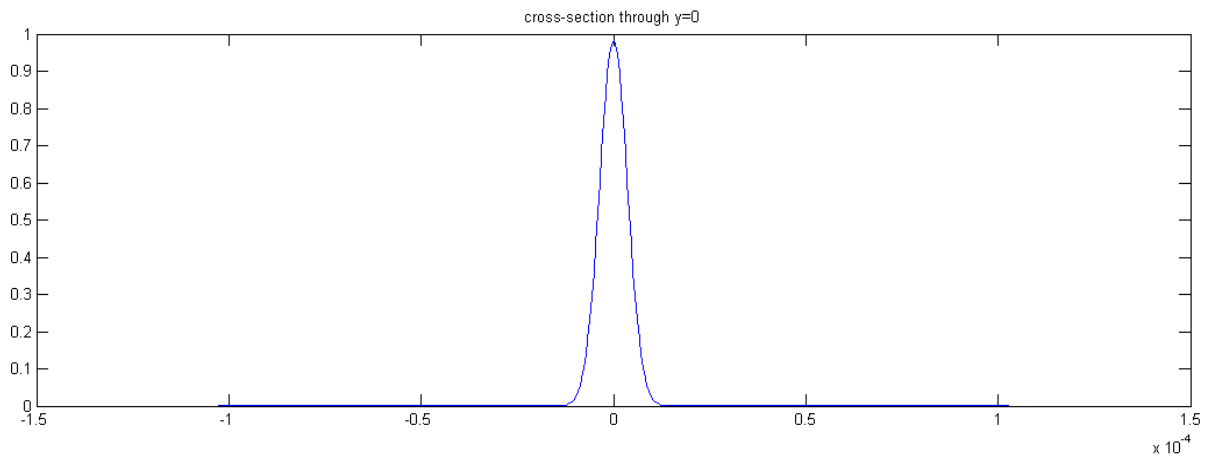

Figure 4. Mean output field amplitude (cross-section through $y=0$, assuming arbitrary units along $x$ and $y$ axis).

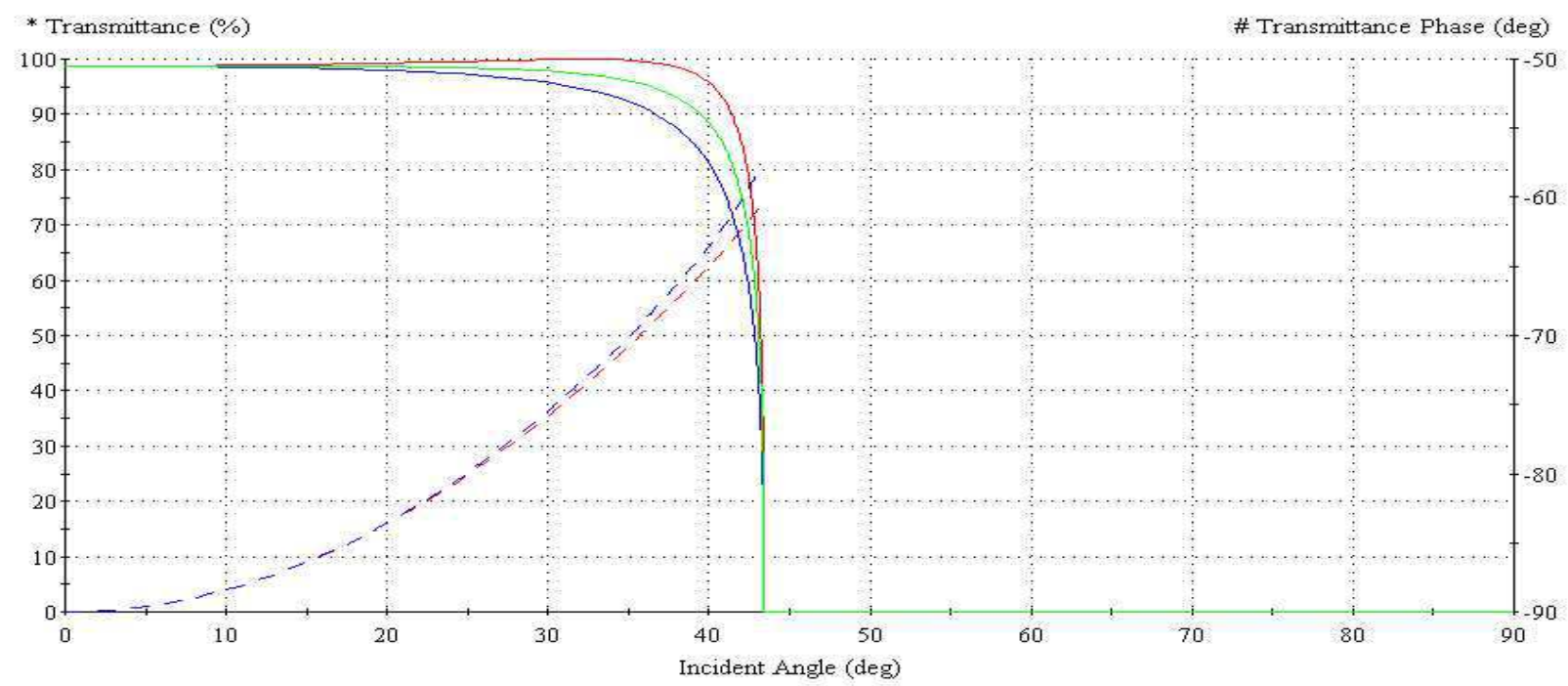

Figure 5. Transmission for all polarisations for a 1 layer coating: magnitude (solid lines) and phase (dashed lines). TM: Red, TE: Blue, Mean: Green.

Table 2. Multilayer coating design parameters for SOS.

\begin{tabular}{llllll}
\hline Layer & Material & Refractive Index & Extinction Coefficient & Optical Thickness & Physical Thickness (nm) \\
\hline Medium & $\mathrm{SOS}$ & 1.45500 & 0.00000 & - & - \\
1 & $\mathrm{Na}_{3} \mathrm{AlF}_{6}$ & 1.35000 & 0.00000 & 0.23865533 & 274.01 \\
2 & $\mathrm{MgF}_{2}$ & 1.37600 & 0.00000 & 0.04876160 & 54.93 \\
3 & $\mathrm{Al}_{2} \mathrm{O}_{3}$ & 1.64974 & 0.00000 & 0.08543824 & 80.27 \\
4 & $\mathrm{MgF}_{2}$ & 1.37600 & 0.00000 & 0.11674291 & 131.51 \\
5 & $\mathrm{Na}_{3} \mathrm{AlF}_{6}$ & 1.35000 & 0.00000 & 0.23325150 & 267.81 \\
Substrate & $\mathrm{Air}$ & 1.00000 & 0.00000 & - & - \\
- & - & - & 0.00000 & 0.72284958 & 808.52 \\
\hline
\end{tabular}

Incident Angle: $0^{0}$; Reference Wavelength: $1550 \mathrm{~nm}$

\subsection{Multilayer Coating}

Next we optimized the transmission with multiple layers (maximum 5) using Optimac. We did not use metals in the coating because metal absorbs the field, nor do we use water because it is impossible to fabricate a liquid coating. We set the targets to a wavelength of $1550 \mathrm{~nm}$, and $100 \%$ transmission for $0^{\circ}-10^{\circ}$ angle. Design details are given in Table 2 . There was almost no reflection of the Gaussian beam when we used this coating. The wave characteristics in multilay- 
ered structure can be analyzed using [9].

Global transmission:

\begin{tabular}{lll}
\hline TM Polarized light & TE Polarized light & Mean \\
\hline 99.9952 & 99.9947 & 99.9949 \\
\hline
\end{tabular}

\section{Conclusion}

Results in general are better for SOS because the refractive index contrast is lower than for SOI. We expected a better performance of the multilayer coating compared to the single layer coating. For SOS this is true, for SOI however, the optimized single layer coating gave the best result. The mean transmission $2.1777 \%$ and $3.4299 \%$ are increased by using single layer and multilayer coating respectively using SOS material system comparing with the reference (without coating).

\section{Acknowledgements}

Authors would like to thank S. Lambert, C.W.Oh and J. B. Martinez for their support.

\section{References}

[1] B.E.A. Saleh, M.C.Teich, Fundamentals of Photonics, Second Edition, Wiley \& Sons, Canada, 2007 \& Microphotonics syllabus, ch.3, session: 2009-2010, www.photonics.intec.ugent.be.

[2] I. Hodgkinson, Q.H. Wu, "Anisotropic antireflection coatings: design and fabrication", OPTICS LETTERS, Vol. 23, No. 19, October 1998, pp. 1553-1555.
[3] H.K.Raut, V.A. Ganesh, A.S. Nair and S. Ramakrishna, "Anti-reflective coatings: A critical, in-depth review", Energy Environ. Sci., 2011, 4, 3779. www.rsc.org/ees.

[4] I.G.Kavakli, K.Kantarli, "Single and double layer antireflection coatings on silicon", Turk J Phys, 26 (2002), pp. 349-354.

[5] I. Khan, "Polarization effect of antireflection coating for SOI material system", International Journal of Science and Research, Vol.1, Issue 3, pp. 59-63.

[6] L. Atternas, L. Thylen, "Single-layer antireflection coating of semiconductor lasers: polarization properties and the influence of the laser structure", Journal of Lightwave Tech., Vol.7, No.2, February 1989, pp.426-430.

[7] R.R.Willey, "Non-polarizing beamsplitter and AR coating design", In Procedings of the Society of Vacuum Coaters on 48th Annual Technical Conference, 2005, ISSN-0737-5921, pp.391-395.

[8] I. Yamada, K.Kintaka, J.Nishii, S.Akioka, Y. Yamagishi and M. Saito, "Transmittance enhancement of a wire-grid polarizer by antireflection coating", APPLIED OPTICS, Vol.48, No.2, January 2009, pp. 316-320.

[9] P. Yeh, Optical Waves in Layered Media, John Wiley and Sons, 1988.

[10] J.A.Dobrowolski, J.E.Ford, B.T.Sullivan, L.Lu and N.R. Osborne, "Conducting antireflection coatings with low polarization dependent loss for telecommunication applications", Optics Express, Vol. 12 Issue.25, 2004, pp. 6258-6269.

[11] H. A. Macleod, Thin-film Optical Filters, 2nd edn, McGraw-Hill, 1989.

[12] www.mellesgriot.com-Technical Information. 